\title{
Dissipation by adhesion hysteresis in dynamic force microscopy
}

\author{
Enrique Sahagún* and Juan José Sáenz ${ }^{\dagger}$ \\ Departamento de Física de la Materia Condensada and Instituto “Nicolás Cabrera," Universidad Autónoma de Madrid, \\ E-28049 Madrid, Spain
}

(Received 9 June 2011; revised manuscript received 7 March 2012; published 6 June 2012)

\begin{abstract}
Power dissipation, as measured in dynamic force microscopy, is usually considered to be proportional to the energy lost in the contact process through the oscillation period. In the presence of adhesion hysteresis, the dissipation is not triggered in each oscillation and only a fraction of the cycles contributes to the dissipated power. We derive a simple analytical closed expression for the dissipated power versus the oscillation amplitude which is in good agreement with the results of numerical simulations. Our theoretical and numerical results predict the existence of two possible dissipation regimes. In the first one, the power dissipated is linear with the energy dissipated by the substrate as expected. In the second regime, a beating pattern appears, causing the fraction of cycles undergoing hysteresis to be inversely proportional to the energy dissipated. As a consequence, the measured power dissipation is a function of a characteristic dissipation length and it is not proportional to the energy lost in a single adhesion process.
\end{abstract}

DOI: 10.1103/PhysRevB.85.235412

PACS number(s): 68.37.Ps, 07.79.Lh, 45.20.dh, 45.20.dg

\section{INTRODUCTION}

Based on the original ideas behind the atomic force microscope (AFM) ${ }^{1}$ it is now possible to measure a large number of physical magnitudes at the nanoscale. ${ }^{2-6}$ A particularly interesting one is the energy dissipated due to tip-sample interaction. $^{7-9}$

There is a vast variety of different processes that give rise to adhesive hysteretic (AH) interactions. ${ }^{8-14}$ These interactions are defined by a change in the force between tip and sample, this change being triggered by the contact of the tip with the sample surface. A common example of $\mathrm{AH}$ interactions is those in which the tip gets attached to the substrate through a physical link: a molecule, a liquid meniscus, or the substrate itself.

Any time the tip activates hysteretic adhesion forces, which henceforth we call an "adhesion event," a certain amount of energy is dissipated. The amount of energy dissipated in each adhesion event depends on the properties of the sample: the hardness of the surface, the energy associated with a chemical contact, the hydrophobicity of the substrate, etc. A direct measure of the energy lost in a single adhesion event $E_{\text {dis }}$ will give unique information about the chemical nature of the sample. ${ }^{14,15}$

In a dynamic AFM experiment, these adhesion events take place in a very short time compared to the oscillation period of the tip and the direct measurement is far from being trivial. Instead of measuring the energy lost in a single oscillation, it is possible to measure the dissipated power $P_{\text {dis }}$ averaged over several oscillations. Considering the time-averaged power balance in the AFM, the time-averaged power lost due to tipsample interactions can be related to the amplitude and phase through 16,17

$$
P_{\mathrm{dis}}=\frac{\omega k}{2 Q}\left[A_{0} A \sin \phi-A^{2} \frac{\omega}{\omega_{0}}\right] .
$$

Here $k$ is the spring constant, $\omega$ the driving angular frequency, $\omega_{0}$ the resonance frequency, $A$ the amplitude, $A_{0}$ the free amplitude, and $Q$ the quality factor.
It could be thought that in a dynamic AFM experiment, where the time scale is well defined through the oscillation period $(T=2 \pi / \omega=1 / f)$, the time-averaged energy dissipated would be simply $P_{\text {dis }}=f E_{\text {dis }}$ (i.e., $E_{\text {dis }}=T P_{\text {dis }}$ ), which implicitly assumes that the tip triggers the dissipative process in each oscillation. However, this is not always the case. After an adhesion event, the cantilever, which has lost energy, will not reach the same amplitude as before the contact, and the tip may not hit the sample surface during the next swings. ${ }^{18}$ Although the mean value of the amplitude, $A$ (averaged over several oscillations), can be well defined, individual oscillations may present small variations in the presence of AH interactions, so that only a certain fraction of the cycles, $v<1$, undergoes the hysteresis. ${ }^{19}$ As a result, the actual time-averaged dissipated power could be significantly lower than expected, i.e.,

$$
P_{\mathrm{dis}}=v f E_{\mathrm{dis}} .
$$

This "beating" regime was confirmed by AFM numerical simulations in the presence of capillary interactions ${ }^{13}$ and also in simulations and experiments on adhesion hysteresis. ${ }^{20}$

Our main goal here is to show that, for a rather general class of $\mathrm{AH}$ interactions, there are two dissipative regimes. There is a high dissipation regime, where $v=1$ and $P_{\text {dis }}$ is proportional to the energy dissipated by the substrate as expected from Eq. (2). Interestingly, we find a low dissipation regime, in which the fraction of cycles undergoing hysteresis process, $v<1$, is inversely proportional to the energy lost in each adhesion hysteretic event, $E_{\text {dis }}$. In this regime, the actual time-averaged dissipated power [Eq. (2)] is a function of a characteristic dissipation length $\lambda_{\text {dis }}$ and the parameters of the cantilever but $P_{\text {dis }}$ does not depend on the actual value of $E_{\text {dis }}$. These two regimes are clearly differentiated in numerical simulations of the AFM dynamics for different $\mathrm{AH}$ interaction forces. Assuming that the phase dynamics is dominated by dissipative interactions, we obtain a closed analytical expression for $P_{\mathrm{dis}}$, as a function of $\lambda_{\text {dis }}$ and the cantilever parameters, which is in excellent agreement with numerical simulations. 


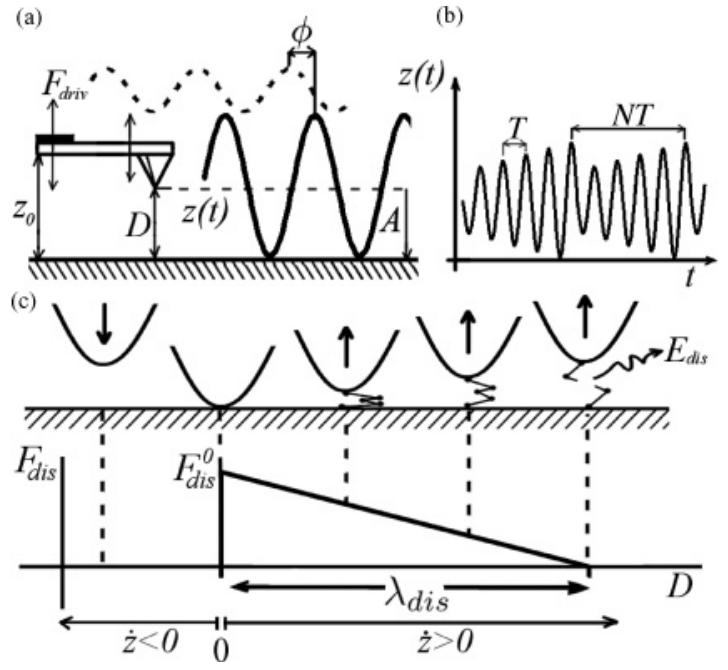

FIG. 1. (a) Sketch illustrating the main AFM parameters entering into the simulation. (b) Trajectory of the tip in the beating regime. The amplitude of the beating is exaggerated. (c) Sketch of the dissipative interaction force $F_{\text {dis }}$. When the tip approaches the sample, $F_{\text {dis }}=$ 0 . After contact, when it moves away $F_{\text {dis }} \neq 0$ and disappears at a characteristic dissipation length $\lambda_{\text {dis }}$.

\section{MODELING THE AFM DYNAMICS}

The usual theoretical approach to modeling AFM dynamics $^{21-24}$ describes the cantilever-substrate system as a driven anharmonic oscillator. Taking into account the cantilever elastic response, the hydrodynamic damping with the medium, and the tip-sample interaction, the equation of motion is given by

$$
\ddot{z}+\frac{\omega_{0}}{Q} \dot{z}+\omega_{0}^{2} z=A_{0} \frac{\omega_{0}^{2}}{Q} \cos (\omega t+\phi)+\frac{\omega_{0}^{2}}{k}\left(F_{\mathrm{ts}}+F_{\mathrm{dis}}\right),
$$

where $A_{0} / Q$ is the amplitude of oscillation of the driving force. $F_{\text {ts }}$ stands for the conservative tip-sample interaction forces, where we include both an attractive van der Waals (vdW) and a repulsive "Hertz plus offset" interaction based on the Derjaguin-Muller-Toporov (DMT) model ${ }^{22,25}$ interaction,

$$
F_{\mathrm{ts}}=\left\{\begin{array}{cl}
-\frac{H R}{D^{2}} & \text { for } \quad D>a_{0}, \\
-\frac{H R}{a_{0}^{2}}+\frac{4}{3} E^{\star} \sqrt{R\left(a_{0}-D\right)^{3}} & \text { for } \quad D \leqslant a_{0},
\end{array}\right.
$$

where $H$ is the Hamaker constant, $R$ is the tip radius, $a_{0}$ is an interatomic distance, $E^{\star}$ the effective Young modulus, and $D=z+z_{0}$ is the tip-sample distance, $z_{0}$ being the distance between the oscillator equilibrium point and the substrate [see Fig. 1(a)]. In Eq. (5), $F_{\text {dis }}$ is the tip-sample dissipative interaction.

\section{A. Adhesive hysteretic interaction forces}

In our analysis of dissipative forces, we restrict ourselves to the important case of $\mathrm{AH}$ interactions. As a rather general approach we assume that, for large enough vibration amplitudes, an $\mathrm{AH}$ force can be written as

$$
F_{\text {dis }}(D)=-\Theta(\dot{z}) F_{\text {dis }}^{0} \mathcal{F}_{\text {dis }}\left(D / \lambda_{\text {dis }}\right),
$$

where $\Theta$ is the Heaviside function.

What makes this interaction nonconservative is the condition on the velocity of the tip $\Theta(\dot{z})$ : It only acts when the tip moves away from the substrate "after contact," i.e., the dissipative interaction is activated only after the tip-sample contact $(D=0)$. The dependence of $F_{\text {dis }}$ on $D$ is contained in the dimensionless dissipation force function $\mathcal{F}_{\text {dis }}\left(D / \lambda_{\text {dis }}\right)$ [with $\left.\mathcal{F}_{\text {dis }}(0)=1\right], \lambda_{\text {dis }}$ being a characteristic interaction length scale. Assuming that the interaction goes to 0 at tip-sample distances much smaller than the amplitude of the cantilever, the energy lost in each contact interaction is simply given by

$$
E_{\text {dis }}=F_{\text {dis }}^{0} \lambda_{\text {dis }} \int_{0}^{\infty} \mathcal{F}_{\text {dis }}(x) d x=c F_{\text {dis }}^{0} \lambda_{\text {dis }},
$$

where $c$ is a number that depends on the interaction law but does not depend on the interaction parameters $\left(F_{\text {dis }}^{0}\right.$ and $\left.\lambda_{\text {dis }}\right)$. As a specific example we can consider a capillary-like interaction of the form

$$
F_{\text {dis }}=F_{\text {dis }}^{0} \times\left\{\begin{array}{cl}
\left(1-D / \lambda_{\text {dis }}\right) \Theta(\dot{z}) & \text { for } 0<D<\lambda_{\text {dis }} \\
0 & \text { otherwise }
\end{array}\right.
$$

where $E_{\text {dis }}=F_{\text {dis }}^{0} \lambda_{\text {dis }} / 2$ (i.e., $c=1 / 2$ ). In terms of the capillary interaction, $\lambda_{\text {dis }}$ can be understood as the length the water meniscus can be stretched until it breaks. [For a graphical representation of Eq. (7), see Fig. 1(c).] This force law is rather general and holds for various types of interactions. An approximately linear drop in the adhesive force was observed in numerical simulations of the breaking processes of atomic-scale contacts where $\lambda_{\text {dis }}$ is of the order of a few interatomic distances (see, e.g., Figs. 3 and 4 in Ref. 10).

\section{B. Numerical simulations of the AFM dynamics}

Once the numerical AFM is set, we simulate a typical AFM experiment with the sample approaching the oscillating tip. The tip starts oscillating far from the substrate. For the sake of simplicity we choose $\omega=\omega_{0}$, so for $z_{0} \gg A, A=A_{0}$ and $\phi=\pi / 2$. Once the oscillator has reached its stationary state, the substrate begins its slow approximation toward the free oscillating lever. Meanwhile the power dissipated is calculated through the measurement of $A$ and $\phi$ using Eq. (1). As in a real experiment, $A$ and $\phi$ are time-averaged values over several oscillations, giving, as a result, a time-averaged $P_{\text {dis }}$. The result obtained for $P_{\text {dis }}$ vs the normalized amplitude $\tilde{A}=A / A_{0}$ is depicted with symbols in Fig. 2(a) for six free amplitudes ${ }^{26}$ It is remarkable that, for a fixed $F_{\text {dis }}$ [Eq. (7) with $F_{\text {dis }}^{0}=10 \mathrm{nN}$ and $\lambda_{\text {dis }}=2 \mathrm{~nm}$, with a fixed amount of dissipated energy per contact $\left(E_{\mathrm{dis}}=10^{-17}\right) \mathrm{J}$, we obtain six different curves for $P_{\text {dis }}$ as a function of $\tilde{A}$. This is obviously in contrast with the naive result, $P_{\text {dis }}=$ constant, predicted for $v=1$ [Eq. (2)].

For low free amplitudes, the curves have a parabolic behavior with a maximun around $\tilde{A} \approx 1 / 2$. For free amplitudes over $20 \mathrm{~nm}$, the curves reach an upper limit which coincides with the maximum dissipated power $E_{\text {dis }} / T=10^{-17} /(100 \mathrm{kHz})^{-1}=$ $1 \mathrm{pW}$. It is clear, then, that in this scenario, $P_{\text {dis }}$ depends not only on $E_{\text {dis }}$ but also on $A$ and $A_{0}$. 

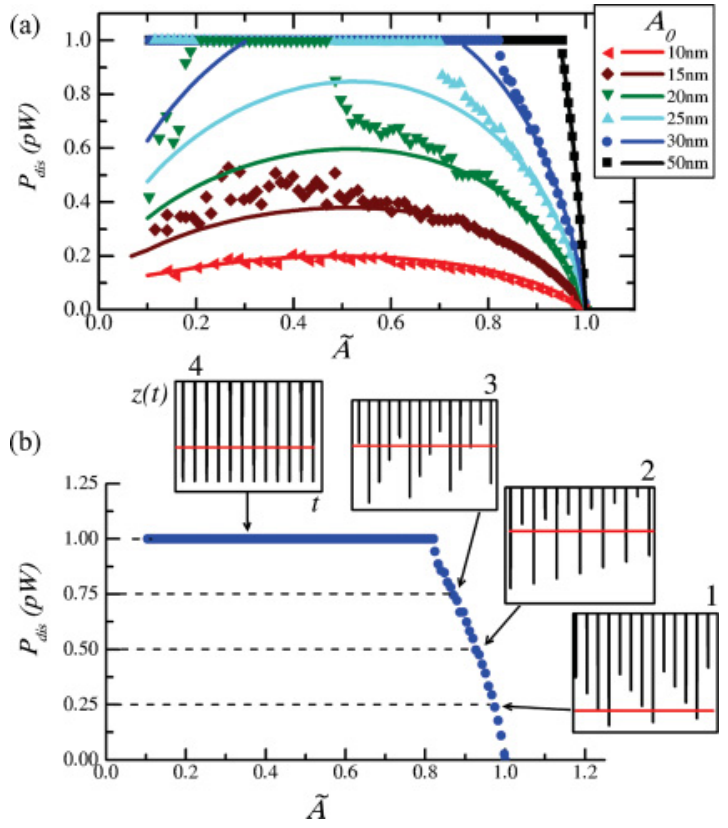

FIG. 2. (Color online) (a) Time-averaged power dissipated vs normalized amplitude $\tilde{A}=A / A_{0}$ for six free amplitudes $A_{0}$ obtained from numerical simulations (symbols). The energy lost in each contact process is $E_{\text {dis }}=10^{-17} \mathrm{~J}\left[\lambda_{\text {dis }}=2 \mathrm{~nm}, F_{\text {dis }}^{0}=10 \mathrm{nN}\right.$; see Eq. (7)]. The maximum dissipated power is $f E_{\text {dis }}=1 \mathrm{pW}$. Solid lines are the analytical predictions based on Eq. (23). (b) Tip trajectories for the $A_{0}=30 \mathrm{~nm}$ curve at four $\tilde{A}$ values. The horizontal (red) line represents the "contact" limit. (b) In (1) there is one dissipative contact interaction every four oscillations $(1 / 4)$. Equivalent curves for (2) $2 / 4$ and (3) (3/4). (4) In the harmonic regime, the dissipative interaction $F_{\text {dis }}$ is activated in each oscillation.

To understand the observed behavior, the trajectory of the tip is depicted as a function of time in Fig. 2(b), 1-4, for a single curve $\left(A_{0}=30 \mathrm{~nm}\right)$. These figures show that the tip does not always reach the dissipation triggering point (red line). The dissipative interaction produces a modulation in the amplitude or "beating" [see Fig. 1(b)]. When the system dissipates one-fourth of the maximum power $\left(E_{\mathrm{dis}} / T\right)$ is just because the tip is activating the dissipative process once in four oscillations [Fig. 2(b), 1], and so on [Figs. 2(b), 2, and 2(b), 3]. Only for certain values of $\tilde{A}$ [see Fig. 2(b), 4] does the tip trigger $F_{\text {dis }}$ in each swing [dissipating the energy predicted for $v=1$ by Eq. (2)], leading to the well-defined plateaus shown in Fig. 2(a).

It can be concluded that the loss of energy that takes place when the tip retracts from the substrate after contact critically affects the motion of the lever. When a tip taps over a substrate in the presence of an $\mathrm{AH}$ interaction, the loss of energy causes a loss of amplitude. If the driving force injects enough power to the oscillator, this amplitude will be recovered in a single oscillation. Then the tip will tap the substrate again, triggering the $\mathrm{AH}$ process in each oscillation [Fig. 2(b), 4]. This is the $v=1$ or high dissipation regime. On the other hand, if the power supplied by the driving force to the lever is not enough, the loss of amplitude is not recovered in the following swim. It takes a certain number of oscillations $N$ for the amplitude to be totally recovered. ${ }^{13,18-20}$ Then the oscillation period of the tip is $T$ but its amplitude is modulated with a frequency $\omega_{b}=2 \pi /(N T)$. During these $N$ oscillations the tip activates the AH interaction just $n$ times, with $v=n / N<1$. Therefore, to make a correct translation of $P_{\text {dis }}$ into $E_{\text {dis }}$, the dynamics of the intermittent contact driven by $v$ has to be unraveled.

\section{ANALYTICAL APPROACH}

Our aim here is to obtain an analytical estimation of the actual beating frequency $\omega_{b}$ and its dependence with the energy loss $E_{\text {dis }}$ and other parameters of the AFM system. Based on the previous discussion, let us assume that the tip trajectory, $z(t)$ [solution of Eq. (3)], is a periodic function with fundamental frequency $\omega_{b}$. We can then expand the trajectory in Fourier series as

$$
z(t)=\sum_{m=1}^{\infty} a_{m} \cos \left(m \omega_{b} t\right)+\sum_{m=1}^{\infty} b_{m} \sin \left(m \omega_{b} t\right),
$$

where $m$ is an integer number. For simplicity we assume that the frequency of the driving force, $\omega$, is an integer multiple of the beating frequency, i.e.,

$$
\omega=\frac{2 \pi}{T}=N \frac{2 \pi}{N T}=N \omega_{b} .
$$

Substituting Eq. (8) into Eq. (3) yields ${ }^{30}$ the following set of coupled equations linking the Fourier coefficients $(m \geqslant 1)$ to the interaction force:

$$
\begin{aligned}
& \left(\omega_{0}^{2}-m^{2} \omega_{b}^{2}\right) a_{m}+m \omega_{b} \frac{\omega_{0}}{Q} b_{m} \\
& =A_{0} \frac{\omega_{0}^{2}}{Q} \cos \phi \delta_{N m}+2 \frac{\omega_{0}^{2}}{k} \frac{1}{N T} \int_{0}^{N T} d t\left\{F_{\mathrm{int}}(z(t)) \cos m \omega_{b} t\right\}
\end{aligned}
$$

$$
\begin{aligned}
& \left(\omega_{0}^{2}-m^{2} \omega_{b}^{2}\right) b_{m}-m \omega_{b} \frac{\omega_{0}}{Q} a_{m} \\
& =-A_{0} \frac{\omega_{0}^{2}}{Q} \sin \phi \delta_{N m}+2 \frac{\omega_{0}^{2}}{k} \frac{1}{N T} \int_{0}^{N T} d t\left\{F_{\mathrm{int}}(z(t)) \sin m \omega_{b} t\right\},
\end{aligned}
$$

where $F_{\text {int }}(z(t)) \equiv F_{\text {ts }}(z(t))+F_{\text {dis }}(z(t))$ is the tip-sample interaction force. This set of equations is equivalent to that discussed by Dürig ${ }^{29}$ but assuming that the frequency of the orbit differs from the driving force frequency. Based on the numerical results, we assume that the interaction is weak in the sense that the dominant frequency is the driving force frequency $\omega$, i.e., all Fourier coefficients with $m \neq N$ are small compared with $a_{N} \equiv A$. If the origin of the time axis is set such that the $N$ coefficient of the sine expansion, $b_{N}=0$, vanishes, the tip-sample distance, at lowest order, is given by

$$
D(t)=z_{0}+z(t) \approx z_{0}+A \cos \omega t,
$$

and substituting in Eqs. (10) and (11), we obtain

$$
\begin{gathered}
\left(\omega_{0}^{2}-\omega^{2}\right) A \approx A_{0} \frac{\omega_{0}^{2}}{Q} \cos \phi+2 \frac{\omega_{0}^{2}}{A k}\left\langle z F_{\mathrm{int}}\right\rangle_{N T} \\
-\omega \frac{\omega_{0}}{Q} A=-A_{0} \frac{\omega_{0}^{2}}{Q} \sin \phi+2 \frac{\omega_{0}^{2}}{A k}\left\langle\dot{z} F_{\mathrm{int}}\right\rangle_{N T},
\end{gathered}
$$


where $\langle\cdots\rangle_{N T}$ stands for the time average over a beating period, $N T$. A first-order correction for other harmonics can be obtained from Eqs. (10) and (11).

The time-averaged power dissipated due to the tip-sample interaction, $P_{\mathrm{dis}}$, is simply given by

$$
P_{\text {dis }}=\left\langle\dot{z} F_{\text {int }}\right\rangle_{N T}=\left\langle\dot{z} F_{\text {dis }}\right\rangle_{N T},
$$

and Eq. (14) is exactly the same as the well-known result ${ }^{16,17}$ given in Eq. (1). Note that conservative interactions do not contribute to the dissipated power since $F_{\text {ts }}(z)$ is symmetric with respect to an extremum of the orbital, while $\dot{z}$ is antisymmetric; i.e., conservative interactions do not contribute to the "sine" equation [Eq. (14)]. For AH interactions, $P_{\text {dis }}$ is given by Eq. (2) since, during the time $N T$, only a certain fraction of the cycles $n / N=v<1$ dissipate energy. Equation (14) can then be rewritten as

$$
\sin \phi=\frac{\omega_{0}}{\omega} \tilde{A}-\frac{1}{\tilde{A}} \frac{v f E_{\mathrm{dis}}}{P_{0}}, \quad P_{0} \equiv \frac{k A_{0}^{2}}{2} \frac{\omega_{0}}{Q} .
$$

Equation (13) can also be written as the well-known result ${ }^{28}$

$$
\cos \phi=Q \tilde{A}\left[1-\frac{\omega^{2}}{\omega_{0}^{2}}-\frac{1}{\tilde{A}^{2}} \frac{\left\langle z F_{\mathrm{int}}\right\rangle_{N T}}{E_{0}}\right], \quad E_{0} \equiv \frac{k A_{0}^{2}}{2} .
$$

Dissipative interactions that depend solely on the velocity are antisymmetric, and as a consequence, they do not contribute to the "cosine" equation, (17). However, AH dissipative interactions, such as those introduced in Eq. (5) have both symmetric and antisymmetric contributions and affect both Eq. (16) and Eq. (17) (see Ref. 29 for a detailed discussion of symmetry arguments). Note that the only difference with respect to the standard approach is that, in the present case, Eqs. (16) and (17) refer to averaged quantities over a time $N T$, instead of a single oscillation $T$. However, this apparently small difference can explain the complex beating phenomena observed in the simulations.

To proceed, we assume that the conservative interaction is relatively weak so we can approximate

$$
\left\langle z F_{\text {int }}\right\rangle_{N T}=\left\langle z F_{\text {ts }}\right\rangle_{N T}+\left\langle z F_{\text {dis }}\right\rangle_{N T} \approx\left\langle z F_{\text {dis }}\right\rangle_{N T} .
$$

Assuming that the AH interaction force is given by Eq. (5) and taking into account that this interaction is activated $n$ times in $N$ oscillations, we have

$$
\begin{aligned}
\left\langle z F_{\mathrm{dis}}\right\rangle_{N T} & \approx v f \int_{T / 2}^{T} z F_{\mathrm{dis}}(D(z)) d t \\
& =v E_{\mathrm{dis}} I\left(A / \lambda_{\mathrm{dis}}, c\right),
\end{aligned}
$$

where we have made use of Eqs. (5) and (6) with $D \approx A+z$ and $\nu f=n /(N T) . I\left(A / \lambda_{\text {dis }}, c\right)$ is a dimensionless factor that depends on the dissipative interaction (through $\lambda_{\text {dis }}$ and $c$ ) but does not depend on $E_{\text {dis }}$ :

$$
I\left(A / \lambda_{\text {dis }}, c\right)=\frac{f}{c} \int_{-A}^{A} \frac{z}{\dot{z}} \mathcal{F}_{\text {dis }}\left(\frac{A+z}{\lambda_{\text {dis }}}\right) \frac{d z}{\lambda_{\text {dis }}} .
$$

This allows us to write Eq. (17) as

$$
\cos \phi \approx Q \tilde{A}\left[1-\frac{\omega^{2}}{\omega_{0}^{2}}-\frac{1}{\tilde{A}^{2}} \frac{\nu E_{\mathrm{dis}}}{E_{0}} I\right] .
$$

Equation (21), together with Eq. (16), is one of the important results of the present work. For the particular case of amplitude-modulated AFM, where the driving frequency $\omega$ is set to the free cantilever resonance frequency $\omega_{0}$, we have

$$
\sin \phi=\tilde{A}-\frac{1}{\tilde{A}} \frac{\nu f E_{\mathrm{dis}}}{P_{0}}, \quad \cos \phi \approx-\frac{Q}{\tilde{A}} \frac{\nu E_{\mathrm{dis}}}{E_{0}} I,
$$

which shows that $v$ must be inversely proportional to $E_{\text {dis }}$. By using a simple trigonometric identity, it is now easy to obtain a closed expression for the fraction of the cycles undergoing dissipation and for the dissipated power, $P_{\mathrm{dis}}=v f E_{\mathrm{dis}}$ (with $v<1)$ :

$$
P_{\mathrm{dis}} \approx P_{0} \tilde{A}^{2}\left\{\frac{1+\sqrt{1+\left(\frac{1}{\tilde{A}^{2}}-1\right)\left[1+(2 \pi I)^{2}\right]}}{\left(1+(2 \pi I)^{2}\right)}\right\} .
$$

This is a most remarkable result since it directly implies that, in the beating regime, $P_{\text {dis }}$ is not proportional to the energy dissipated in each $\mathrm{AH}$ event but it is a relatively complex function of the characteristic length $\lambda_{\text {dis }}$. In other words, for a general $\mathrm{AH}$ force law, increasing the force constant $F_{\text {dis }}^{0}$ but keeping $\lambda_{\text {dis }}$ constant should give approximately the same value of $P_{\mathrm{dis}}$, even though in each contact process the energy lost increases. This is a general result in the beating regime independent of the particular form of the dissipative adhesion forces.

\section{DISCUSSION AND CONCLUDING REMARKS}

It is of interest to check the analytical results against the numerical simulations. For the particular case of capillary interactions [linear adhesion forces, e.g., Eq. (7)], it is easy to obtain an explicit expression for the dimensionless factor $I$ :

$$
I=-\frac{A}{2 \pi \lambda_{\mathrm{dis}}} \sqrt{\frac{2 A}{\lambda_{\mathrm{dis}}}-1}\left[\frac{\lambda_{\mathrm{dis}}}{A}-1+\sqrt{\frac{2 A}{2 A-\lambda_{\mathrm{dis}}}}\right]
$$

for $A<A_{0}$. Comparison between the simple analytical approach and the numerical results is illustrated in Fig. 2, where we have plotted $P_{\text {dis }}$ vs $\tilde{A}$ for a given value of $E_{\text {dis }}$. The agreement between numerical results for $P_{\text {dis }}$ (symbols) and Eq. (23) (lines), except for relatively small $\tilde{A}$ values, is excellent (without any adjustable parameter). Note that the numerical simulation includes both conservative and dissipative tip-sample interactions ( $F_{\text {ts }}$ and $\left.F_{\text {dis }}\right)$. Equations (23) and (24) thus provide a good description of power dissipation despite the simplicity of our approximation.

In Fig. 3 we plot $P_{\text {dis }}$ vs $E_{\text {dis }}$ at a fixed amplitude $\tilde{A}=0.9$. Different curves are computed by increasing $E_{\text {dis }}=F_{\text {dis }}^{0} \lambda_{\text {dis }} / 2$ at a fixed $\lambda_{\text {dis }}\left(\lambda_{\text {dis }}=1,2\right.$, and $\left.4 \mathrm{~nm}\right)$ or at a fixed $F_{\text {dis }}^{0}$ (2, 5, and $10 \mathrm{nN})$. Symbols are the results of numerical simulations in the beating regime and solid lines correspond to Eq. (23). The dashed (gray) line corresponds to Eq. (2) with $v=1$, which represents the maximum amount of power the system can dissipate. The first thing observed is that equal values of $E_{\mathrm{dis}}$ produce different values of $P_{\mathrm{dis}}$. As predicted by our analytical approach, the dissipated power at fixed $\lambda_{\text {dis }}$ remains constant and independent of the energy lost in each contact process. Again, the agreement between theory and the numerical simulation is remarkable.

In summary, the analysis of the dynamics of a driven oscillator in the presence of a $\mathrm{AH}$ interaction reveals two 


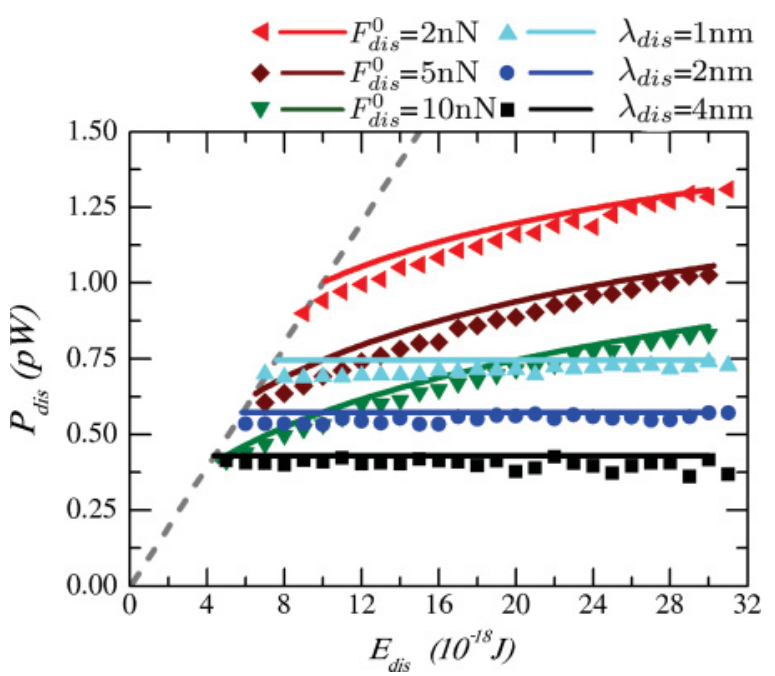

FIG. 3. (Color online) Power dissipated vs energy lost in each contact process. Different curves are obtained by increasing $E_{\text {dis }}$ ( $E_{\text {dis }}=F_{\text {dis }}^{0} \lambda_{\text {dis }} / 2$ ) at fixed $\lambda_{\text {dis }}$ or fixed $F_{\text {dis }}^{0}$ values (top). Symbols correspond to numerical results, and solid lines to the analytical approximation, Eq. (23). The straight dashed line indicates the position of $E_{\mathrm{dis}} / T$.

different dissipation regimes. In the first one, the power dissipated is proportional to the energy dissipated per contact, $E_{\text {dis }}$, throughout the oscillation period. In the other, the beating of the oscillator dominates the rate at which energy is lost. The power dissipated is not linear with $E_{\text {dis }}$ but is a function of the characteristic dissipation length $\lambda_{\text {dis }}$ as well as of the parameters of the cantilever. This result stresses the fact that the translation between power and energy in this scenario is not trivial.

Our results are supported by both numerical simulations of the AFM dynamics and a simplified analytical approach based on two major approximations. First, we assumed that the interaction forces were weak in the sense that the oscillatory motion of the tip was dominated by the Fourier component corresponding to the driving frequency. The second major approximation was to assume that the conservative part of the interaction was smaller than the dissipative part. Despite the simplicity of our approach, we found an excellent agreement between our analytical closed expression for the dissipated power and the results of numerical simulations. Taking into account our rather general assumptions and the scalability of the equations, we believe that similar behavior should be observed in a large variety of mechanical systems and devices of interest from nano to macro technological applications.

\section{ACKNOWLEDGMENTS}

We thank S. Albaladejo, M. Fuchs, P. Garcia-Mochales, M. Köber, M. Luna, R. Pérez, A. Rahman, I. Suárez, N. de Sousa, and L. S. Froufe for interesting discussions. This work was supported by the Spanish MICINN Plan Nacional (Grant No. FIS2009-13430-C02-02) and by the Comunidad de Madrid Microseres-CM (Grant No. S2009/TIC-1476). *enrique.sahagun@uam.es

${ }^{\dagger}$ http://www.uam.es/mole

${ }^{1}$ G. Binnig, C. F. Quate, and Ch. Gerber, Phys. Rev. Lett. 56, 930 (1986).

${ }^{2}$ C. Stroh et al., Biophys. J. 87, 1981 (2004).

${ }^{3}$ M. Jaafar et al., Ultramicroscopy 109, 693 (2009).

${ }^{4}$ R. W. Carpick and M. Salmeron, Chem. Rev. 97, 1163 (1997).

${ }^{5}$ L Fumagalli et al., Nanotechnology 17, 4581 (2006).

${ }^{6}$ R. Merkel, P. Nassoy, A. Leung, K. Ritchie, and E. Evans, Nature 397, 50 (1999).

${ }^{7}$ P. Martin, S. Marsaudon, J. P. Aimé, and B. Bennetau, Nanotechnology 16, 901 (2005).

${ }^{8}$ P. D. Ashby and C. M. Lieber, J. Am. Chem. Soc. 127, 6814 (2007).

${ }^{9}$ R. García, R. Magerle, and R. Pérez, Nat. Mater. 6, 405 (2007).

${ }^{10}$ U. Landman et al., Science 248, 454 (1990).

${ }^{11}$ N. Oyabu, P. Pou, Y. Sugimoto, P. Jelinek, M. Abe, S. Morita,

R. Perez, and O. Custance, Phys. Rev. Lett. 96, 106101 (2006).

${ }^{12}$ N. F. Martínez and R. García, Nanotechnology 17, 167 (2006).

${ }^{13}$ E. Sahagún, P. García-Mochales, G. M. Sacha, and J. J. Sáenz, Phys. Rev. Lett. 98, 176106 (2007).

${ }^{14}$ Y. Sugimoto et al., Nature 446, 64 (2007).

${ }^{15} \mathrm{X}$. Xu, J. Melcher, S. Basak, R. Reifenberger, and A. Raman, Phys. Rev. Lett. 102, 060801 (2009).
${ }^{16}$ J. P. Cleveland, B. Anczykowski, A. E. Schmid, and V. B. Elings, Appl. Phys. Lett. 72, 2613 (1998).

${ }^{17}$ J. Tamayo and R. García, Appl. Phys. Lett. 73, 2926 (1998).

${ }^{18}$ S. J. T. Van Noort et al., Ultramicroscopy 69, 117 (1997).

${ }^{19}$ B. Gotsmann and H. Fuchs, Appl. Surf. Sci. 188, 355 (2002).

${ }^{20}$ M. Köber, E. Sahagún, M. Fuss, F. Briones, M. Luna, and J. J. Sáenz, Phys. Status Solidi (RRL) 2, 138 (2008).

${ }^{21}$ R. García and R. Pérez, Surf. Sci. Rep. 47, 197 (2002).

${ }^{22}$ R. García and A. San Paulo, Phys. Rev. B 60, 4961 (1999).

${ }^{23}$ N. A. Burham et al., Nanotechnology 8, 67 (1997).

${ }^{24}$ B. Gotsmann and H. Fuchs, Appl. Phys. A 72, S55 (2001); B. Gotsmann, C. Seidel, B. Anczykowski, and H. Fuchs, Phys. Rev. B 60, 11051 (1999).

${ }^{25}$ B. V. Derjaguin, V. M. Muller, and Y. P. Toporov, J. Colloid Interface Sci. 53, 314 (1975).

${ }^{26}$ The values used in the numerical integration are $\omega_{0}=6.28 \times 10^{5}$, $R=10 \mathrm{~nm}, k=5 \mathrm{~N} / \mathrm{m}, Q=100, H=2.0 \times 10^{-20} \mathrm{~J}, E^{\star}=$ $50 \mathrm{GPa}$, and $a_{0}=0.2 \mathrm{~nm}$.

${ }^{27}$ L. Nony, R. Boisgard, and J. P. Aimé, J. Chem. Phys. 111, 1615 (1999).

${ }^{28}$ A. San Paulo and R. García, Phys. Rev. B 64, 193411 (2001).

${ }^{29}$ U. Dürig, New J. Phys. 2, 5 (2000).

${ }^{30}$ Alternatively, we could obtain the same set of equations invoking a least-action principle as discussed in Refs. 7, 27 and 29. 